\title{
The tumor microenvironment: the involvement of the selectin-selectin-ligand axis in tumor-endothelium interactions Isaac P Witz*
}

Address: Department of Cell Research and Immunology, George S. Wise Faculty of Life Sciences, Tel Aviv University, Tel Aviv, Israel

* Corresponding author

from 2006 International Meeting of The Institute of Human Virology

Baltimore, USA. 17-2I November, 2006

Published: 21 December 2006

Retrovirology 2006, 3(SuppI I):S9I doi:I0.1I86/I742-4690-3-SI-S9I

(c) 2006 Witz; licensee BioMed Central Ltd.

Tissue-specific leukocyte localization is a requirement for immune surveillance and plays a key role in the pathogenesis of various inflammatory diseases. Leukocyte adhesion to endothelial cells is crucial for their extravasation to sites of inflammation. Extravasating leukocytes migrate through the endothelial barrier by first adhering to the endothelial cell surface and then transmigrating through the endothelial cell layer. Adhesion to the endothelium usually requires a cascade of steps mediated by the selectins, leukocyte activating chemotactic factors, and endothelial activating proinflammatory cytokines as well as by integrins. These molecules act in concert and regulate the sequence of distinct steps.

In order for cancer cells to form metastases at specific organ sites, pre metastatic cells must exit the vasculature, migrate into these sites and be able to survive and proliferate there. Molecules involved in transendothelial migration are therefore key players in metastasis formation.

Does the extravasation of pre-metastatic tumor cells from blood vessels follow the pathway of leukocyte extravasation? The molecular basis of cancer cell extravasation is poorly understood. A comprehensive and thorough understanding of the mechanisms underlying these processes is an absolute requirement for the development of therapeutic modalities aimed to block or retard metastasis.

This presentation is focused on the interactions between endothelial (E)-selectin with its ligands on colorectal cancer (CRC) cells. Fucosylated selectin ligands initiate the interaction between extravasating cells and endothelium via their binding to endothelial selectins. We will discuss 2 distinct aspects of such an interaction. The first is the regulation of CRC adhesive capacity to endothelial cells and CRC extravasation. We will demonstrate that the fucose-generating FX enzyme plays a key role in the biosynthesis of selectin ligands and in their interaction with selectins. The second aspect is the regulation of gene expression in CRC by signals delivered to such cells by Eselectin. 